\title{
Designing Digital Experiences in an Analog World
}

\author{
Max Zabramny \\ Organic, Inc. \\ 1375 Broadway, New York, NY 10018 USA \\ zabramny@gmail.com
}

\begin{abstract}
Designing intuitive, digital experiences in today's fast-paced world of ever evolving interfaces, requires a designer that's in touch with the root of human physical interactions.
\end{abstract}

Digital, Analog, Interfaces, Experience Design, Inspiration

\section{INTRODUCTION}

Put your mouse down, close your laptop, and step away from your iPhone. If you truly want to create great digital interfaces, than you must look for inspiration outside the medium in which you are designing. As designers, we're curious by nature about what will come next, and how we can use today's technology to push our ideas about tomorrow. But as human beings, we have a distinct connection to the tactile rather than the abstract, and the emotional rather than the inanimate. It is in these principles that digital interaction designers should be rooted. However, the latest onslaught of new products and digital advances has created an environment where these connections are being severed. This paper aims to provide a set of guidelines and principles that can be used to design digital interfaces that are based on the manner in which human beings interact with everyday physical objects.

\section{PERPETUAL COMPLEXITY}

We live in a world that's become less and less analog. We've become a race that's moving closer to digitizing everything from our musical selection, to what we had for breakfast that morning. Yet in this stroke of genius, this blast of ones and zeros, we've become more and more detached from each other. It's ignorant to suggest that the resolution to this issue is to limit the exposure to our daily digital interactions. However, perhaps the answer lies in our jobs as interaction designers, to create digital services and experiences that are closer in behavior to the physical world that our users inhabit.

\section{A RETURN TO SIMPLICITY}

One way to return to a more intuitive and human sense of digital interaction design is to look to our siblings in other design disciplines for inspiration. One such discipline is industrial design. Industrial designers spend much of their time observing how people use things; their attention to detail is paramount. The main reason for this is that the end deliverable of industrial design is a tangible product that can be held in one's hand, creating a palpable and emotional connection. With a digital interface, the connection isn't as direct; usually because there are physical boundaries in the way of the user and the object being manipulated, such as keyboards, mice, and the physical space produced by these devices. Even with a touch screen interface, the screen itself creates a degree of separation between the user and the locus of attention. Unintuitive design further expands this separation by relying on unfamiliar visual analogies in the interface.

One fundamental flaw that causes this is the concept of misinformed inspiration. The constant evolution of new functionality and design produces an expectation in the designer that they should consider these evolved interfaces as the foundation of their design thinking. However, no matter how elaborate or advanced, these interfaces only drive the designer further away from core human interaction principles. To solve this problem, the designer should look to the affordances that people assign to every day objects as part of the basic building blocks of human-object interaction. Buttons, levers, hinges, springs, and other physical interfaces provide the foundation of our daily tactility. Therefore, we as designers must be cognizant of the muscle memory that is attached to 
them by our users, and attempt to translate it to the experiences we create. At the same time, the graphical manifestation of their digital counterparts must never stray too far from the contextual goals of the user. You can find examples of this methodology scattered across your daily digital path. We need only to consider the surplus of web and desktop interfaces that use modal windows to direct the user to information that is currently most crucial. Or the recent rise of tagging digital content in order to easily retrieve it later, creating familiarity in a new object by paring it with a term that holds a previously assigned definition. In both examples, designers have created a methodology for building mental models that are based on everyday human actions - such as stacking papers in order of importance or using post-it notes as reminders.

However, focusing strictly on industrial design as a single point of inspiration isn't enough; the modern designer must go further, consulting disciplines that may, at first glance, seem disconnected from the design field. One such area of study is modern day animated filmmaking. While the concept of storytelling and narrative is nothing new, the advances in these areas by companies such as Pixar has created new methodologies of storytelling that relate directly to the field of interaction design. Specifically, Pixar has been able to successfully anthropomorphize everyday objects and bring a sense of personality and behavior to them, without breaking the paradigms of how they work or how we're used to using them. The same concept can be applied to interaction design when creating digital interfaces. Introducing a sense of motion and anthropomorphic reaction such as bouncing, shaking, and scaling can create a strong connection with the user; providing an element of empathy that ultimately leads to a stronger relationship with the device and brand at hand. Apple's operating systems (OS) for both, desktop and the iPhone, use these principles successfully when providing the user with affordances and alerts. Consider the iPhone OS, in which, icons begin to shake when inferring their ability to be rearranged. Or the desktop OS login window, which rapidly moves side to side when the user enters an incorrect password, simulating a human headshake.

Encapsulating these guidelines together means approaching the user experience strictly from a visceral perspective, a kind of cognitive ergonomics. It's a process where choice and engagement on the part of the user is an extremely highly regarded action. Will your user choose to engage with the interface that has been presented in front of them? Will they understand how to convert their goals into actions? Or, will the sheer amount of "creativity" simply confuse them? Ultimately, the actions we all take when using any type of interface, be it physical or digital, say something about who we are and how we see the world around us. As designers, it's important to keep this fresh in our minds, because no matter how much we improve and upgrade the devices around us, we can't upgrade our minds. And so we must continuously return to what grounds us as human beings - the physical world around us, and how we use the blocks that it's made up of. 